\title{
EI Rol Predisponente del Optimismo: Hacia un Modelo Etiológico del Bienestar
}

\author{
The causal role of the optimism; towards an etiological model of well-being.
}

\author{
Pablo Vera-Villarroel \\ Paula Pávez \\ Universidad de Santiago de Chile, USACH, Chile \\ $\&$ \\ Jaime Silva \\ Universidad de la Frontera, Chile
}

(Rec:10 de febrero de 2012 / Acep: 11 de mayo de 2012)

\begin{abstract}
Resumen
En los últimos años, la investigación de los recursos psicológicos que favorecen la salud y el bienestar han experimentado un aumento considerable. Recientes datos muestran a partir de estudios altamente rigurosos y controlados, algunos de los beneficios que producen los estados y afectos positivos, como también del optimismo y el bienestar, entre otros estados positivos en la salud física y psicológica. A pesar de esta evidencia, existe aún poca información de los mecanismos y variables relacionadas en los procesos de bienestar, optimismo y afectos positivos. Así mismo existe escaso consenso acerca de los mecanismos psicológicos y/o neurofisiológicos que expliquen la relación con los estados positivos y su beneficio para la salud y principalmente acerca de las variables causales o etiológicas del bienestar.

Se revisa la evidencia científica acerca de los beneficios de los estados positivos como el optimismo, felicidad y principalmente el bienestar. Se propone un modelo causal conceptual que plantea que el nivel de bienestar subjetivo y psicológico, está influenciado previamente por el nivel de optimismo. De esta forma y basada en la literatura y evidencia previa se plantea que el optimismo, al ser una condición aprendida desde la niñez, favorecería la mayor cantidad de experiencias positivas y emociones positivas. Estos estados afectivos positivos mediarían la relación entre el optimismo y el bienestar y sus consecuencias.
\end{abstract}

Palabras clave: Optimismo, bienestar, modelo etiológico.

\begin{abstract}
Research into the psychological resources that benefit health and well-being have increased dramatically in recent years. The latest data from highly rigorous and controlled studies show some of the benefits that produce positive states and affects, as well as optimism and well-being, among other positive states in physical and psychological health. In spite of this evidence, there is little information about the mechanisms and variables related to the processes of well-being, optimism and positive affects. In addition, there is no real consensus regarding the psychological and/or neurophysiological mechanisms that explain the relation to positive states and their benefit for health and mainly with respect to the causal or etiological variables of well-being. The scientific evidence about the benefits of positive states like optimism, happiness and mainly well-being is reviewed. A conceptual causal model is proposed which suggests that the level of subjective and psychological well-being is previously influenced by the level of optimism. This way, and based on the prior literature and evidence, it is proposed that optimism, being a condition learned from childhood, may stimulate the greatest number of positive experiences and positive emotions. These positive affect states would then mediate the relation between optimism and well-being and their consequences.
\end{abstract}

Keywords: Optimism, well-being, etiological model.

Este manuscrito fue realizado gracias a los aportes del proyecto FONDECYT No 1110520.

Correspondencia: Dr. Pablo Vera-Villarroel, Escuela de Psicología. Universidad de Santiago de Chile, USACH. Avenida Ecuador 3650 , $3^{\circ}$ Piso. Santiago. Email: pablo.vera@usach.cl. 


\section{Introducción}

La Psicología Clínica tradicionalmente ha estudiado al hombre dentro de la perspectiva de la psicopatología o enfermedad mental (Vera, 2006), ya sea en sus determinantes o consecuencias (Greenglas \& Fiksenbaum, 2009). Lo anterior, es esperable si se piensa que el objetivo tradicional de la psicología clínica ha sido el alivio del sufrimiento humano, subestimándose con esto el estudio y la comprensión de otros fenómenos del comportamiento humano, que contribuyen a su felicidad, aumento de fortalezas, virtudes y su bienestar general (Martínez, 2006; Martorelli \& Mustaca, 2004). Si bien estas variables siempre han estado presentes en la historia de la Psicología, solo últimamente se han destinado esfuerzos a su estudio científico (Vázquez, 2006; Vázquez, Hervás \& Ho, 2006).

En los últimos años, la investigación de los recursos psicológicos que favorecen la salud y el bienestar ha experimentado un aumento considerable (Remor, Amorós \& Carboles, 2006; Vecina, 2006), evidenciando una tendencia al afrontamiento de variables positivas y preventivas en lugar de aspectos negativos y patológicos (Contreras \& Esguerra, 2006; Grau, Hernández $\&$ Vera-Villarroel, 2005). En este marco, se ha desarrollado una línea de investigación sobre los afectos positivos, recursos y fortalezas del ser humano (Martínez, 2006), como de su comportamiento individual y grupal. Recientes datos muestran a partir de estudios altamente rigurosos y controlados, algunos de los beneficios que producen los estados y afectos positivos (Lyubomirsky, King \& Diener, 2005).

Dentro de esta perspectiva, nace la Psicología Positiva, cuyo objetivo es estudiar cómo la ausencia de características positivas, su promoción y las circunstancias de la vida pueden estar implicadas en una variedad de trastornos mentales (Wood \& Joseph, 2010). Esta idea no es nueva dentro de la psicología, sin embargo el énfasis en el estudio riguroso y científico de estas variables, procesos y estados positivos es lo que ha caracterizado a este movimiento. Este surgimiento parece relevante si se considera la evidencia recogida en los últimos años como las investigaciones que han descubierto relaciones entre enfermedades tan importantes como el cáncer con variables como el optimismo, o esta misma variable con la salud física y psicológica (Mustaca, Kamenetzky \& Vera-Villarroel, 2010; Ortiz, Ramos \& Vera-Villarroel, 2003; Vera-Villarroel, 2005; Wilhelm, Wedguood, Parker, Geerligs \& Hadzi-Pavlovic, 2010); optimismo y estrés postraumático (Vera-Villarroel, Zych, Celis-Atenas, Córdova-Rubio \& BuelaCasal, 2011); optimismo y mejores habilidades de resolución de problemas sociales (Vera-Villarroel \& Guerrero, 2003); felicidad con esperanza de vida y salud física (Koopmans, Geleijnse, Zitman \& Giltay, 2010); felicidad y estilos de vida saludables en universitarios (Piqueras, Kuhne, Vera-Villarroel, Van Straten \& Cuijpers, 2011); bienestar y actividades diarias (Meléndez, Tomas \& Navarro, 2011); bienestar subjetivo con esperanza de vida (Xu \& Roberts, 2010); bienestar psicológico con mejor rendimiento en el trabajo, relaciones de éxito, salud, longevidad, felicidad y niveles de positividad (Avey, Luthans, Smith \& Palmer, 2010; Díaz, Flores \& Soromaa, 2011); bienestar y satisfacción marital (Scorsolini-Comin \& Dos Santos, 2011); emociones y actitudes positivas con presión arterial, mejora en la capacidad funcional en pacientes con insuficiencia cardíaca (Davidson, Mostofsky \& Whang, 2010); mejora en el equilibrio hormonal y menores niveles de lípidos, cáncer de mama, accidentes cerebrovasculares y depresión (Marrero \& Carballeira, 2006; Xu \& Roberts, 2010), entre otros.

A pesar de la evidencia señalada anteriormente, según Davidson et al. (2010), existe aún poca información de los mecanismos y variables relacionadas en los procesos de felicidad, optimismo y afectos positivos, como escaso consenso acerca de los mecanismos psicológicos y/o fisiológicos en los estados positivos. El panorama descrito puede derivar de la naturaleza reciente del campo de estudio de la psicología positiva; si bien en una primera fase, se ha promovido y destacado la relevancia de su estudio, recién nos encontramos en la fase de obtención de pruebas acerca de los beneficios de estos estados. Y aun cuando se han dado importantes pasos en este último punto, resta por comprender la totalidad de los datos en formulaciones teóricas integradoras. Un ejemplo de ello, es la mayor disposición de conocimiento en cuanto a los beneficios que producen los afectos y estados positivos por separado, que la relación entre estos estados en conjunto y su interacción.

\section{La psicología positiva: Definición del campo de estudio y sus problemas irresueltos}

Teóricamente, los conceptos que se incluyen en el ámbito de estudio de la Psicología Positiva, son el bienestar, satisfacción, optimismo, felicidad, entre otros. Respecto del término bienestar, este ha sido correlacionado de manera directa con distintas variables como esperanza de vida, salud, depresión, ansiedad, etc. (Avey et al., 2010; Marrero \& Carballeira, 2010; Seligman, Steen, Park \& Peterson 2005; Xu \& Roberts, 2010), sin embargo ha evidenciado problemas en su definición al existir numerosas formas de interpretación (Ryan \& Deci, 2001). De acuerdo a ello, en los últimos años se ha visto una cristalización de este constructo y nuevas ideas que aunque surgen desde otras líneas de investigación claramente son complementarias con la psicología positiva, de este modo van surgiendo así dos grandes aproximaciones de bienestar; el subjetivo y el psicológico (Keyes, Shmotkin \& Ryff, 2002; Ryan \& Deci, 2001), o también llamadas la escuela hedónica y la escuela eudaimónica. La primera se centra en las experiencias subjetivas de las personas, incluyendo variables de personalidad y respuestas emocionales, dando lugar al constructo bienestar subjetivo. Éste a su vez incluye dos componentes principales; los afectos (presencia de emociones positivas y ausencia de emociones negativas) y la satisfacción con la vida 
(elemento cognitivo) (Rodríguez-Carvajal, Díaz-Méndez, Moreno-Jiménez, Blanco-Abarca \& Van Dierendonck, 2010; Rodríguez-Fernández \& Goni-Grandmontagne, 2011). Existen estudios que relacionan la presencia de esta variable con la salud, longevidad, redes sociales (Xu \& Roberts, 2010), depresión y apoyo social percibido (Mella, González, D'Appolonio, Maldonado, Fuenzalida \& Díaz, 2004). La segunda escuela, hace referencia al bienestar psicológico de las personas, centrando su atención en el desarrollo de capacidades y crecimiento personal, concebidas ambas como principales indicadores del funcionamiento positivo (Díaz, Rodríguez-Carvajal, Blanco, Moreno-Jiménez, Gallardo, Valle \& Van Dierendonck, 2006). Sus primeras concepciones se articularon en torno a conceptos como autorrealización (Maslow, 1968), funcionamiento pleno (Rogers, 1961), madurez (Allport, 1961), teorías sobre la salud mental positiva (Johada, 1958) y teorías sobre el ciclo vital (Erikson, 1959).

Por otra parte, Ryff (1989) propuso un modelo integral y multidimensional para el estudio del bienestar psicológico (Rodríguez-Carvajal et al., 2010; Vázquez et al., 2006), formulando seis dimensiones, reiteradas con posterioridad (Ryff \& Keyes, 1995), en que la teoría subyacente dividía al bienestar en: auto-aceptación, relaciones positivas con los otros, dominio del ambiente, autonomía, crecimiento personal y propósito en la vida (Tomas, Meléndez, Oliver, Navarro \& Zaragoza, 2010).

Recientemente se ha dado a conocer a la comunidad científica un estudio que establece que la ausencia de bienestar psicológico constituye un factor de riesgo para la depresión, independientemente de la presencia de factores negativos y de una salud física alterada, siendo las personas con bajo bienestar psicológico 7,16 veces más propensas a estar deprimidas 10 años más tarde. Lo anterior se corrobora, incluso después de controlar la personalidad, el funcionamiento negativo, depresión previa, características demográficas, económicas, físicas y variables de salud (Wood \& Joseph, 2010). Esto es muy importante ya que, si bien tradicionalmente se ha relacionado el bienestar (específicamente su ausencia) con la depresión, el estudio mencionado demuestra que el aspecto crítico (factor de riesgo para depresión) es la ausencia o bajo nivel de bienestar. No obstante, una de las críticas a este estudio está dada por el hecho de que la mayor parte de la investigación fue transversal, planteando la posibilidad de que la reducción del bienestar psicológico sea consecuencia de la depresión, más que estar implicado en la etiología del trastorno.

En esta misma línea de evidencia científica se ha informado recientemente que el apoyo social y el optimismo son dos variables capaces de predecir un aumento en los estados postraumáticos de sobrevivientes a trasplantes nueve años después. Ello apoyaría además la idea de que estos constructos tienen un rol aún más importante que ser sólo variables relacionadas con estados positivos o negativos (Tallman, Shaw, Schultz \& Altmaier, 2010).

Sin embargo, a pesar del beneficio descubierto por estas investigaciones acerca de las variables positivas, la evidencia actual no ha estado alejada del cuestionamiento y valoración crítica por grupos de especialistas. Algunos de estos cuestionamientos han estado centrado en aspectos conceptuales, teóricos y supuestas ignorancias de la historia de la psicología (para una reciente revisión en este aspecto ver Fernández-Ríos \& Novo, 2012). Por otra parte, y en una línea de análisis diferente sustentada principalmente a partir de estudios previos y datos empíricos, también se ha criticado el valor positivo por sí mismo de ciertas variables que desde la Psicología Positiva se buscaría fomentar, específicamente McNulty \& Fincham (2012) muestran evidencias que los rasgos psicológicos y procesos no serían intrínsecamente positivos o negativos, sino más bien sus implicaciones para el bienestar dependen de las circunstancias en las cuales ellos funcionan.

Adicionalmente, es probable que las críticas más interesantes para el avance de esta línea de investigación hayan surgido de los análisis críticos de los resultados y hallazgos, especialmente desde el ámbito de la Salud. Una muestra de ello es el número especial en la revista Annals of Behavioral Medicine (Aspinwal \& Tedeschi, 2010a; Aspinwal \& Tedeschi, 2010b; Coyne, Tennen \& Ranchor, 2010; Gorin, 2010; Miller, Sherman \& Christensen, 2010) que expone sobre el valor de la Psicología Positiva en la salud. En este número, si bien se presenta variada evidencia a favor, igualmente se expone otra aproximación, como la planteada por Coyne, que entre otras cosas afirma que los estudios no cuentan con datos que puedan demostrar la relación causal entre las variables positivas y la salud. Es decir, existiría evidencia a favor del valor predictivo de estos constructos para una buena salud, pero esto no explica el porqué de esta predicción y por tanto el valor causal sería modesto. Estableciéndose con ello la necesidad de mayor evidencia causal y/o etiológica de las variables positivas para la salud (Coyne \& Tennen, 2010).

\section{Neurociencia del Bienestar}

El estudio neurocientífico de las emociones o neurociencia afectiva (Davidson, 1998) ha circulado principalmente por la vía de la comprensión de los estados afectivos negativos, intentando develar su rol en diferentes clases de alteraciones psicopatológicas (Silva, 2003). Este sesgo, tal como se ha descrito en secciones anteriores, no es privativo de la neurociencia, y sus disciplinas afines, sino que ha caracterizado el estudio de la conducta humana bajo los más diversos prismas teóricos y empíricos. En años recientes, se han reportado algunos pocos trabajos que permiten comenzar a delinear lo que podría describirse como una neurociencia del bienestar y de las experiencias positivas en general, incluyendo el optimismo. 
Dentro de este marco científico incipiente, la revisión de Burgdorf \& Panksepp (2006) puede considerarse como un esfuerzo fundacional para la comprensión de las experiencias positivas desde la perspectiva neurobiológica. Analizando la literatura científica disponible, los autores concluyen que la afectividad positiva posee una representación cerebral, principalmente subcortical y conectado al sistema dopaminérgico. Se destaca en el análisis el rol del estriado ventral, tanto en la anticipación del reforzamiento (activación) como en la respuesta frente a la presencia del reforzamiento (desactivación). Así mismo, Burgdorf \& Panksepp destacan el rol de la amígdala en el afecto positivo, mostrando consistentemente una desactivación en numerosas experiencias positivas y diversos procedimientos experimentales. Aun cuando los aspectos subcorticales son claves para la generación de la afectividad negativa, también se reconoce el rol de la corteza, particularmente la corteza orbitofrontal (activación) frente a estímulos que generan experiencias positivas.

El optimismo, por otro lado, ha sido muy poco estudiado en el ámbito de la neurociencia. Hasta la fecha, al menos dos reportes permiten identificar algunos aspectos distintivos de procesos psicológicos asociados al optimismo. El primero de ellos (Sharot, Riccardi, Raio \& Phelps, 2007) analizó cómo el sesgo optimista (acentuar las consecuencias positivas de un evento futuro por sobre las negativas) fue mediado por determinados mecanismos neurales. El hallazgo principal muestra que el sesgo optimista se asociaba a una reducción de la activación de la amígdala y de la corteza cingulada anterior derecha, estructuras relacionadas con la representación de consecuencias negativas de la conducta. Una segunda investigación (Sharot, Korn \& Dolan, 2011), develó que las personas más optimistas mostraban niveles inferiores de activación en regiones del córtex prefrontal (giro pre-frontal inferior derecho) asociados al mantenimiento en memoria de información desafiante de información previa.

En suma, la neurociencia afectiva ha tenido muy poco que decir sobre la psicología positiva, y especialmente sobre el optimismo. Esta falta de información representa un desafío para los investigadores, siendo un campo urgente de promover y desarrollar.

\section{Un propuesta de solución:}

A pesar de las cuestiones pendientes en cuanto al rol causal para el bienestar, existe evidencia teórica y empírica que puede entregar ciertas hipótesis dignas de verificar. En este sentido se cuenta con evidencia que mostraría al optimismo como variable que puede predecir los niveles de bienestar (Augusto-Landa, Pulido-Martos \& López-Zafra, 2011). En este mismo sentido, Lechn (2010) propone una posible respuesta a estas preguntas basándose en la teoría de autorregulación (Carver \& Scheir, 1990). Según este modelo, las personas se esfuerzan en la medida que ven sus objetivos como alcanzables, logrando así experimentar resultados positivos y, por el contrario, cuando las personas aprecian los resultados como inalcanzables se retiran de sus objetivos y experimentan emociones negativas. Lechn establece una relación estrecha entre optimismo y los objetivos que la gente enfrenta todos los días, proponiendo el posible rol mediador de las emociones negativas y los objetivos diarios específicos, entre el optimismo y una mejor salud metal y física. De esta forma, demostró en un estudio realizado con estudiantes universitarios, evidencia de que las variables psicológicas pueden mediar el beneficio que el optimismo presentaría en la salud de los sujetos. Sin embargo, a pesar de esta reciente evidencia, el autor trata indistintamente emociones con expectativas negativas (o positivas) y analiza directamente los posibles factores mediadores entre el optimismo y sus consecuencias. Asimismo, no incorpora explícitamente en su estudio variables de bienestar (psicológico o subjetivo) a pesar de ser constructos estrechamente relacionados, con igual cantidad de evidencia en cuanto a sus beneficios. Adicionalmente y al igual que la mayoría de los estudios previos, reconoce parcialmente las diferentes variables psicológicas positivas en las que se ha encontrado evidencia de su beneficio. De esta forma, el autor no estima que el bienestar podría considerarse indirectamente por la ausencia de estados negativos, como la mayoría de los estudios previos.

Teniendo esto presente y con respecto al concepto de bienestar, existen al menos tres aspectos que aún no se encontrarían totalmente resueltos en la literatura actual y que es necesario atender. Un primer punto de discusión respecto del término bienestar, lo encontramos en una crítica, basada en la visión excesivamente etnocéntrica del constructo, y por reflejar perspectivas propias de las sociedades individualistas contemporáneas, basadas en la noción de "éxito" (Rodríguez-Carvajal et al., 2010).

En segundo lugar, ambos modelos de bienestar no son concluyentes en sus definiciones, ya que en algunos estudios es considerado sólo como presencia de felicidad (afecto positivo) y ausencia de depresión y ansiedad (afecto negativo) (Karademas, 2006), mientras que en otros el afecto negativo es definido de acuerdo a características de personalidad (Ho, Cheung \& Cheung, 2010). Además, los estudios han tratado indistintamente los diferentes conceptos de bienestar, no existiendo evidencia de que en un mismo trabajo se hayan considerado ambos modelos en la salud. Este punto es central para el avance y despliegue de investigaciones que permitan un mejor desarrollo científico y aplicado de la Psicología Positiva ${ }^{1}$.

\footnotetext{
1 Ante las dificultades descritas, un concepto surgido del estudio científico actual de la psicología positiva que podría ofrecer una vía de soluciones metodológicas y teóricas, es el concepto de felicidad, que ha sido considerado en algunas ocasiones indistintamente con el concepto de bienestar (Avey et al., 2010; Diener et al., 2003; Karademas, 2006; Lyubomirsky, King \& Diener, 2005; Seligman, 1998). La felicidad podría responder a las preguntas antes mencionadas, por ser un estado considerado recientemente como aprendido (Lyubomirsky, 2008; Seligman,
} 


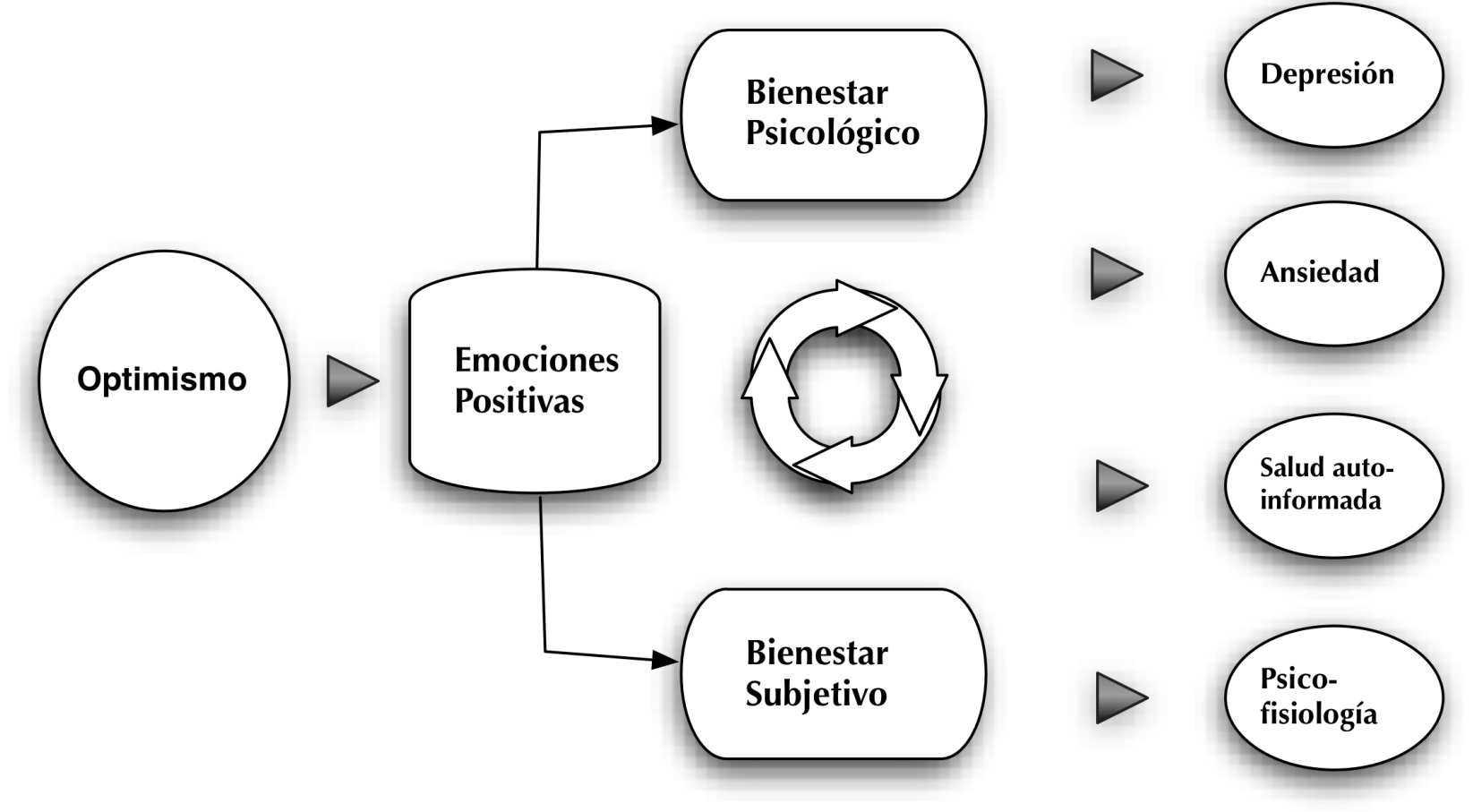

Figura 1. Modelo Conceptual etiológico del Bienestar y sus Consecuencias

En tercer lugar, el bienestar es un concepto complejo que alude al óptimo funcionamiento psicológico y experiencial de las personas (Ryan \& Dici, 2001). Ante esto, surge la pregunta de cuáles son los determinantes del nivel de bienestar en un momento dado, o qué hace que un sujeto evalúe su nivel de bienestar de una determinada manera en un momento específico. En este sentido, existe considerable evidencia que demuestra que las variables económicas y culturales pueden influir (Chang \& Asakawa, 2003; Chang, Asakawa \& Sanna, 2001; Diener, Oishi \& Lucas, 2003; Morrison, Tay \& Diener, 2011; Tay \& Diener, 2011; Vera-Villarroel et al., 2012), así como las circunstancias de la vida (control de la temperatura, alimentación, salud, control ambiental, relaciones sociales), metas y valores de las personas (Diener et al., 2003). Sin embargo, también existe evidencia de que esta contribución no es total, y que su participación es hasta cierto nivel, a partir del cual su influencia decrece. Por otra parte, debido al relativismo

1998, 2003). En este sentido los seres humanos podrían ejercitarse para ser felices o menos infelices, como ejemplo, se cuenta con ejercicios $\mathrm{y}$ actividades cotidianas que han demostrado que el sujeto aumenta su nivel de felicidad (Diener \& Biswas-Diener, 2008; Lyubomirsky, 2008). Sin embargo, la felicidad es un concepto limitado, pues hace referencia a percepciones, análisis, o significados de la experiencia humana del presente. De acuerdo a Díaz et al. (2006), es un balance de los afectos positivos y negativos que provoca la experiencia inmediata. $\mathrm{Y}$ al igual que en el caso del bienestar constituye una evaluación global de la experiencia del sujeto en un momento determinado. cultural y diferencias intersubjetivas de las personas (intercambio de afecto positivo por otras cosas que los sujetos valoran como positivas), los teóricos del bienestar se encuentran con fuertes variaciones y discrepancias (Diener et al., 2003; Ryan \& Deci, 2001).

\section{El optimismo: Una propuesta de investigación para} la comprensión del Bienestar y sus consecuencias positivas.

Tal como se revisó en la sección anterior el estado del campo de estudio no permite actualmente responder la pregunta de qué es lo que hace que el sujeto llegue en un momento dado a realizar una determinada evaluación global de la felicidad y el bienestar. Se considera que la respuesta a esta interrogante aún no ha sido saldada ni enfrentada desde los diversos estudios de la Psicología Positiva.

Frente a esto, nuestra propuesta sostiene que el optimismo (Seligman, 1998) sería un factor clave para la conformación de un cierto nivel de bienestar y felicidad. Además el concepto de optimismo no tiene límites en el tiempo como el concepto de felicidad, pues su definición hace mención a expectativas positivas acerca de la ocurrencia de eventos positivos futuros (Carver, Sheier \& Sagerstrom, 2010). En otras palabras, tiene que ver con una percepción o expectativa generalizada por parte de las personas, de que el futuro depara cosas positivas y no necesariamente tiene que ver con un análisis global de la vida en el momento 
actual (Vera-Villarroel, Córdova-Rubio \& Celis-Atenas, 2009). Adicionalmente dada la concepción del optimismo como una expectativa o estilo de pensamiento, éste se fundamenta en un estado aprendido (Avia \& Vásquez, 1999; Seligman, 1998), a diferencia del bienestar, constructo del que no tenemos evidencia actual acerca de su aprendizaje. Se propone entonces que el optimismo, como una condición aprendida, sería capaz de determinar o predisponer un alto o bajo nivel de bienestar futuro (Augusto-Landa et al., 2011; Avey et al., 2010; Carver et al., 2010; Ho et al., 2010; Marrero \& Carballeira, 2010; Martínez, 2006). En este sentido nuestra propuesta plantea al optimismo como un factor independiente y predisponente para el bienestar. Además, si se toma en cuenta que la depresión y la ansiedad han demostrado estar igualmente correlacionadas con el optimismo (Abdel-Khalek, 2006; Brydon, Walker, Wawrzyniak, Chart \& Steptoc, 2009; Chico \& Ferrando, 2008; De Moor, De Moor, Basen-Engquist, Kundelka, Bevers \& Cohen, 2006; Kivimaki, Elovainio, Singh-Manoux, Vahtera, Helenius \& Pentti, 2005; Lobel, DeVincent, Kaminer \& Meyer, 2000; Puskar, Ren, Bernardo, Haley \& Stark, 2008; Remor et al., 2006, Vecina, 2006; Vera, 2006), éste podría constituir un factor independiente y predisponente para el bienestar (tanto para el subjetivo como psicológico) y de riesgo para la depresión y la ansiedad como la salud en general (VeraVillarroel, 2005).

Se propone que desde un punto de vista etiológico e integrador operarían los factores biológicos como factores predisponentes, a partir de los cuales los factores psicológicos en el ser humano operarían los procesos de aprendizaje, modelamiento y socialización (ver figura 1).

Existe considerable evidencia de que el optimismo seria un estado psicológico aprendido. Nuestra propuesta establece que este aspecto es instruido desde los primeros años de vida, en los cuales los cuidadores, familia, pares $\mathrm{y}$ colegios moldean las expectativas, formas de pensar y de actuar que configurarían una predisposición pesimista u optimista (la descripción o categoría solo es en un sentido académico ya que es muy probable que esto más que ser dos aspectos o estados sea más bien un continuo). Considerando el aspecto aprendido del optimismo desde la niñez se puede plantar que este estado y sus beneficios serían adecuados predictores de un bienestar en la adultez, que es cuando la mayoría de las investigaciones se han centrado en analizar, especialmente en los beneficios del bienestar pero en población adulta. En este sentido, existe evidencia de que determinadas variables positivas en la niñez serían predictoras de un bienestar positivo en la adultez tal como lo han demostrado recientemente Richards \& Huppert (2011).

La propuesta teórica planteada, a partir de la evidencia empírica expuesta, sugiere que el nivel de bienestar tanto subjetivo como psicológico, que determinan los estados emocionales y neurofisiológicos, estarían influenciados previamente por el nivel de optimismo, variable factible de aprendizaje, como por la mediación de afectos positivos. En este sentido el optimismo sería la variable causal principal que influiría en mayor cantidad de experiencias positivas y por lo tanto de emociones positivas a lo largo de la vida. El experimentar mayor cantidad de afectos positivos a través del tiempo y esencialmente a través de muchos años determinaría finalmente un mejor bienestar posterior. En este sentido los afectos positivos mediarían la relación entre el optimismo y el bienestar y sus consecuencias dada la condición previa optimista. Esta formulación instaura una aproximación a un modelo etiológico y causal respecto al bienestar, que incluye una propuesta de explicación acerca de los mecanismos y variables relacionadas en estos procesos, así como de los beneficios que producen los estados $\mathrm{y}$ afectos positivos en conjunto.

Se considera que estos planteamientos teóricos y empíricos aportan datos para la superación de la falencia planteada por Davison et al. (2010), quienes señalan que hasta la fecha existiría falta de información acerca de los mecanismos y variables relacionadas con el optimismo y el bienestar. Estos aportes permitirán determinar el efecto mediador de ciertas variables, contribuyendo a una mejor comprensión del efecto indirecto del optimismo sobre el bienestar.

Además, el hecho de incluir un mayor número de variables mediadoras, estableciendo una asociación predictorcriterio en más de una ocasión, contribuiría, según los planteamientos de Frazier, Tix \& Barron (2004) para lograr formular modelos causales propiamente tales. Es decir, se considera que futuros estudios deberían establecer un mayor número de variables como mediadoras que expliquen el aprendizaje del optimismo y su relación causal con el bienestar.

\section{Referencias}

Abdel-Khalek, A. (2006). Measuring happiness with a single-item scale. Social Behavior and Personality, 34, 139-150.

Allport, G.W. (1961). Pattern and growth in personality. New York: Holt, Rinehart, \& Winston.

Aspinwal, L.G., \& Tedeschi, R.G. (2010a). The value of positive psychology for health psychology: Progress and pitfalls in examining the relation of positive phenomena to health. Annals of Behavioral Medicine, 39, 4-15.

Aspinwall, L.G., \& Tedeschi, R.G. (2010b). Of babies and bathwater: A reply to Coyne and Tennen's views on positive psychology and health. Annals of Behavioral Medicine, 39, 27-34.

Augusto-Landa, J.M., Pulido-Martos, M., \& López-Zafra, E. (2011). Does perceived emotional intelligence and optimism/pessimism predict psychological well-being?. Journal of Happiness Studies, 12, 463-474.

Avia, M., \& Vásquez, C. (1999). Optimismo inteligente. Madrid: Alianza.

Avey, J., Luthans, F., Smith, S., \& Palmer, N. (2010). Impact of positive psychological capital on employee well-being over time. Jounal of Occupatinal Health Psychology, 15, 17-28.

Brydon, L., Walker, C., Wawrzyniak, A.J., Chart, H., \& Syeptoc, A. (2009). Dispositional optimism and stress-induced changes in immunity and negative mood. Behavioral and Inmunity, 6, 810-816.

Burgdorf, J., \& Panksepp, J. (2006). The neurobiology of positive emotions. Neuroscience \& Biobehavioral Reviews, 30, 173-187. 
Carver, C. S., \& Scheier, M. (1990). Principles of self-regulation: Action and emotion. In T. E. Higgins, \& R. M. Sorrentino (Eds.), Handbook of motivation and cognition: Foundations of social behavior (pp. 3-52). New York: Guilford Press.

Carver, C., Scheier, M., \& Segerstrom, S. (2010). Optimism. Clinical Psychology Review, 30, 879-889.

Chang, E.C., Asakawa, K., \& Sanna, L.J. (2001). Cultural variations in optimistic and pessimistic bias: Do easterners really expect the worst and westerners really expect the best when predicting future life events? Journal of Personality and Social Psychology, 81,476-491.

Chang, E.C., \& Asakawa, K. (2003). Cultural variations on optimistic and pessimistic bias for self versus a sibling: Is there evidence for self-enhancement in the west and for self-criticism in the east when the referent group is specified? Journal of Personality and Social Psychology, 84, 569-581.

Chico, E., \& Ferrando, P. (2008). Variables cognitivas y afectivas como predoctoras de satisfacción en la vida. Psicothema, 20, 408-412.

Contreras, F., \& Esguerra, G. (2006). Psicología positiva: Una nueva perspectiva en psicología. Diversitas. Perspectivas en Psicología, 2, 311-319.

Coyne, J.C., Tennen, H., \& Ranchor, A.V. (2010). Positive psychology in cancer care: A story line resistant to evidence. Annals of Behavioral Medicine, 39, 35-42.

Coyne, J.C., \& Tennen, H. (2010). Positive psychology in cancer care: Bad science, exaggerated claims, and unproven medicine. Annals of Behavioral Medicine, 39, 16-26.

Davidson, K.W., Mostofsky, E., \& Whang, W. (2010). Don't worry, be happy: Positive affect and reduced 10-year incident coronary heart disease: The Canadian Nova Scotia Health Survey. European Heart Journal, 9, 1065-1070.

Davidson, R. J. (1998). Affective style and affective disorders: Perspectives from affective neuroscience. Cognition and Emotion, 12, 307-330.

De Moor, J.S., De Moor, C.A., Basen-Engquist, K., Kudelka, A., Bevers, M.W., \& Cohen, L. (2006). Optimism, distress, health-related quality of life, and change in cancer antigen 125 among patients with ovarian cancer undergoing chemotherapy. Psychosomatic Medicine, 4, 555-562.

Díaz, E.M, Flores, M.E., \& Soromaa, H. (2011). Fiabilidad y validez de constructo del test munsh para medir felicidad, en población de adultos mayores chilenos. Universitas Psychologica, 10, 567-580.

Díaz, D., Rodríguez-Carvajal, R., Blanco, A., Moreno-Jiménez, B., Gallardo, I., Valle, C., \& Van Dierendonk, D. (2006). Adaptación española de las escalas de bienestar psicológico de Ryff, Psicothema, 18, 572-577.

Diener, E., \& Biswas-Diener, R. (2008). Hapiness. Unlocking the mysteries of psychological wealth. Oxford: Blackwell Publishing.

Diener, Ed., Oishi, S., \& Lucas, R. (2003). Personality, culture, and wellbeing: Emotional and cognitive evaluations of life. Annual Review of Psychology, 54, 403-425.

Erikson, E. (1959). Identity and the life cycle. Psychological Issues. 1, 18-164.

Fernandez-Rios, L., \& Novo, M. (2012). Positive pychology: Zeigeist (or spirit of the times) or ignorance (or disinformation) of history? International Journal of Clinical and Health Psychology, 12, 333-346.

Frazier, P. A., Tix, A. P., \& Barron, K.E. (2004). Testing moderator and mediator effects in counseling psychology research. Journal of Counseling Psychology, 51, 115-134.

Grau, J., Hernández, E., \& Vera- Villarroel, P. E. (2005). Estrés, salutogénesis y vulnerabilidad. En E. Hernández, \& J. Grau (Ed.), Psicología de la salud: Fundamentos, metodología aplicaciones. (pag. 113-177). Guadalajara: Universidad de Guadalajara.

Gorin, S.S. (2010). Theory, measurement, and controversy in positive psycholology, health psychology, and cancer: Basics and next steps. Annals of Behavioral Medicine, 39, 43-47.

Greenglas, E., \& Fiksenbaum, L. (2009). Proactive coping, positive affect, and well-being. Testing for mediation using path analysis. European Psychologist, 14, 29-39.

Ho, M., Cheung, F., \& Cheung, S. (2010). The role of meaning in life and optimism in promoting well-being. Personality and Individual Differences, 48, 658-663.
Johada, M. (1958). Current concepts of positive mental health. New York: Basic Books

Karademas, E. (2006). Self-efficacy, social support and well-being. The mediating role of optimism. Personality and Individual Differences, 40, 1281-1290.

Keyes, C., Shmotkin, D., \& Ryff, C. (2002). Optimizing well-being: The empirical encounter of two tradictions. Journal of Personality and Social Psychology, 82, 1007-1022.

Kivimaki, M., Elovainio, M., Singh-Manoux, A., Vahtera, J., Helenius, H., \& Pentti, J. (2005). Optimism and pessimism as predictors of change in health after death or onset of severe illness in family. Health Psychology, 24, 413-421.

Koopmans, T., Geleijnse, J., Zitman, F., \& Giltay, E. (2010). Efeccts of happiness on all-cause mortality during 15 years of follow-up: The arnhem elderly stud. Journal of Happiness Studies, 11, 113-124.

Lench, H. (2010). Personality and health outcomes: Making positive expectations a reality. Journal of Happiness Studies, 12, 493-507.

Lobel, M., DeVincent, C., Kaminer, A., \& Meyer, B. (2000). The impact of prenatal maternal stress and optimistic disposition on birth outcomes in medically high-risk women. Health Psychology, 19, 544-553.

Lyubomirsky, S. (2008). La ciencia de la felicidad. Barcelona. Uranos

Lyubomirsky, S., King, L., \& Diener, E. (2005). The benefits of frecuent positive affect: Does happiness lead to success? Psychological Bulletin, $131,803-855$.

Martinez, M. (2006). El estudio científico de las fortalezas trascendentales desde la psicología positiva. Clínica y Salud, 17, 245-258.

Martorelli, A., \& Mustaca, A. (2004). Psicología positiva, salud y enfermos renales crónicos. Revista Nefrología, Diálisis y Trasplante, 24, 99-104.

Maslow, A.H. (1968). Toward a psychology of being (2nd ed). New York: Van Nostrand.

Marrero, R., \& Caballeira, M. (2006). El papel del optimismo y el apoyo social en el bienestar subjetivo. Salud Mental, 33, 39-46.

McNulty, J.K., \& Fincham, F.D. (2012). Beyond positive psychology? toward a contextual view of psychological processes and well-being. American Psychologist, 67, 101-110.

Melendez, J.C., Tomas, J.M., \& Navarro, E. (2011). Everyday life activities and well-being: Their relationships with age and gender in the elderly. Anales de Psicologia, 27, 164-169.

Mella, R., González, L., D’Appolonio, J., Maldonado, I., Fuenzalida, A. \& Díaz, A. (2004). Factores asociados al bienestar subjetivo en el adulto mayor. Psyche, 13, 79-89.

Miller, S.M., Sherman, A.C., \& Christensen, A.J. (2010). Introduction to special series: The great debate - evaluating the health implications of positive psychology. Annals of Behavioral Medicine, 39. 1-3.

Morrison, M., Tay, L., \& Diener, E (2011). Subjective well-being and national satisfaction: Findings from a worldwide survey. Psychological Science, 22, 166-171.

Mustaca, A.E., Kamenetzky, G., \& Vera-Villarroel, P. (2010). Relations between positive and negative variables in a sample of argentine students. Revista Argentina de Clínica Psicológica, 19, 227-235.

Ortiz, J., Ramos, N., \& Vera-Villarroel, P.E. (2003). Optimismo y salud: Estado actual e implicaciones para la Psicología Clínica y de la Salud. Suma Psicológica, 10, 119-134

Piqueras, J.A., Kuhne, W., Vera-Villarroel, P., Van Straten, A., \& Cuijpers, P (2011). Happiness and health behaviours in Chilean college students: A cross-sectional survey. BMC Public Health, 11,443.

Puskar, K., Ren, D., Bernardo, L.M., Haley, T., \& Stark, KH. (2008). Anger correlated with psychosocial variables in rural youth. Issues in Comprehensive Pediatric Nursing, 3, 71-87.

Remor, E., Amorós, M., \& Carboles, J. (2006). El optimismo y la experiencia de ira en relación con el malestar físico. Anales de psicología, $22,37-44$

Richards, M., \& Huppert, F.A. (2011). Do positive children become positive adults? Evidence from a longitudinal birth cohort study. Journal of Positive Psychology, 6, 75-87.

Rodríguez-Carvajal, R., Díaz-Méndez, D., Moreno-Jiménez, B., BlancoAbarca, A., \& Van Dierendonck, D. (2010). Vitalidad y recursos internos como componentes del constructor de bienestar psicológico. Psicothema, 22, 63-70. 
Rodríguez-Fernández, A., \& Goni-Grandmontagne, A. (2011). The threedimensional structure of subjective wellbeing. Anales de Psicología, 27, 327-332.

Rogers, C.R. (1961). On becoming a person. Boston: Houghton Mifflin.

Ryan, R., \& Deci, E. (2001). On happiness and human potentials: A review of research on hedonic and eudaimonic well-being. Annual Review of Psychology, 52, 141-166.

Ryff, C.D. (1989). Beyong Ponce de Leon and life satisfacción: New directions in quest of successful aging. International Journal of Behavioral Development, 12, 35-55.

Ryff, C., \& Keyes, C. (1995). The structure of psychological well-being revisted. Journal of Personality and Social Psychology, 69, 719-727.

Scorsolini-Comin, F., \& Dos Santos, M.A. (2011). Relations between subjective well-being and marital satisfaction on the approach of positive psychology. Psicologia-Reflexao e Critica, 24, 658-665.

Seligman, M.E.P. (1998). Aprenda optimismo. Barcelona: Grijalbo.

Seligman, M.E.P. (2003). La auténtica felicidad. Buenos Aires: Vergara.

Seligman, M. E. P., Steen, T. A., Park, N., \& Peterson, C. (2005). Positive psychology progress empirical validation of interventions. American Psychologist, 60, 410-421.

Sharot, T., Korn, C.W., \& Dolan, R.J. (2011). How unrealistic optimism is maintained in the face of reality. Nature Neuroscience, 14, 1475-1479.

Sharot, T., Riccardi, A.M., Raio, C.M., \& Phelps, E.A. (2007). Neural mechanisms mediating optimism bias. Nature, 450, 102-106.

Silva, J. (2003). Biología de la regulación emocional: Su impacto en la psicología del afecto y la psicoterapia. Terapia Psicológica, 21, 163-172.

Tallman, B., Shaw, K., Schultz, J., \& Altmaier, E. (2010). Well-Being and posttraumatic growth in unrelated donor marrow transplant survivors: A nine-year longitudinal study. Rehabilitation Psychology. 55, 204-210.

Tay, L., \& Diener, E. (2011). Needs and subjective well-being around the world. Journal of Personality and Social Psychology, 101, 354-365.

Tomas, J., Meléndez, J., Oliver, A., Navarro, E., \& Zaragoza, G. (2010). Efectos del método en la escala de Ryff: Un estudio en población de personas mayores. Psicológica, 31, 383-400.

Vázquez, C. (2006). La psicología positiva en perspectiva. Papeles del Psicólogo, 27, 1-2.
Vázquez, C., Hervás, G., \& Ho, S. (2006). Intervenciones clínicas basadas en la psicología positiva: Fundamentos y aplicaciones. Psicología Conductual, 14, 401-432.

Vecina, M. (2006). Emociones positivas. Papeles de Psicólogo, 27, 9-17. Vera, B. (2006). Psicología Positiva: Una nueva forma de entender la Psicología. Papeles del Psicólogo, 27, 3-8.

Vera-Villarroel, P.E. (2005). Optimismo y salud. En L. Florez-Alarcón, M.M. Botero, \& B. Moreno-Jiménez (Eds.), Psicología de la salud. (pp. 77-89). Bogota ALAPSA- Universidad del Norte.

Vera-Villarroel, P., Celis - Atenas, K., Pavez, P., Lillo, S., Bello, F., Díaz, N., \& López, W. (2012). Money, age and happiness: Association of wellbeing with socio-demographic variables. Revista Latinoamericana de Psicología, 44, (en prensa).

Vera-Villarroel, P., Córdova-Rubio, N., \& Celis-Atenas, K. (2009). Evaluación del optimismo: un análisis preliminar del Life Orientation Test versión revisada (LOT-R) en población chilena. Universitas Psicológica, 8, 61-68.

Vera-Villarroel, P.E., \& Guerrero, A. (2003). Diferencias en habilidades de resolución de problemas sociales en sujetos optimistas y pesimistas: un estudio descriptivo. Universitas Psychologica, 2, 21-26.

Vera-Villarroel, P., Zych, I., Celis-Atenas, K., Córdova-Rubio, N., \& Buela-Casal, G (2011). Chilean validation of the posttraumatic stress disorder checklist-civilian version (PCL-C) after the earthquake on february 27, 2010. Psychological Reports, 109, 47-58.

Wilhelm, K., Wedguood, L., Parker, G., Geerligs, L., \& Hadzi-Pavlovic, D. (2010). Predicting mental health and well-being in adulthood. Journal of Nervous and Mental Disorders, 2, 85-90.

Wood, A., \& Joseph, S. (2010). The absence of positive psychological (eudemonic) well-being as risk factor for depression: A ten years cohorte stydy. Journal of Affective Disorders, 122, 213-217.

Xu, J., \& Roberts, R. (2010). The power of positive emotions: It's a matter of life or death - subjective well-being and longevity over 28 years in a general population. Health Psychology, 29, 9-19. 